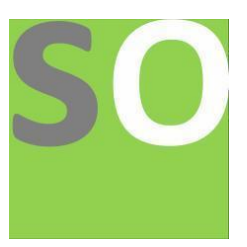

Article title: Performance Investigation of Hydraulic Actuator Based Mass Lift System using MPC and LQR Controllers Authors: mustefa jibril[1], Messay Tadese[2], Eliyas Alemayehu[3]

Affiliations: School of Electrical and Computer Engineering[1], Faculty of Electrical and Computer Engineering[2]

Orcid ids: 0000-0002-3165-2410[1]

Contact e-mail: mustefazinet1981@gmail.com

License information: This work has been published open access under Creative Commons Attribution License http://creativecommons.org/licenses/by/4.0/, which permits unrestricted use, distribution, and reproduction in any medium, provided the original work is properly cited. Conditions, terms of use and publishing policy can be found at https://www.scienceopen.com/.

Preprint statement: This article is a preprint and has not been peer-reviewed, under consideration and submitted to ScienceOpen Preprints for open peer review.

DOI: 10.14293/S2199-1006.1.SOR-.PPPPCGZ.v1

Preprint first posted online: 31 May 2020

Keywords: Hydraulic actuator, Model predictive control, MPC, linear quadratic regulator, LQR 


\title{
Performance Investigation of Hydraulic Actuator Based Mass Lift System using MPC and LQR Controllers
}

\author{
Mustefa Jibril $^{1}$, Messay Tadese ${ }^{2}$, Eliyas Alemayehu Tadese ${ }^{3}$ \\ ${ }^{1}$ Msc, School of Electrical \& Computer Engineering, Dire Dawa Institute of Technology, Dire Dawa, \\ Ethiopia \\ ${ }^{2}$ Msc, School of Electrical \& Computer Engineering, Dire Dawa Institute of Technology, Dire Dawa, \\ Ethiopia \\ ${ }^{3}$ Msc, Faculty of Electrical \& Computer Engineering, Jimma Institute of Technology, Jimma, Ethiopia
}

\begin{abstract}
A hydraulic actuator is a system that can provide a large power amplification in industries and factories. In this paper, mass lifter hydraulic actuator system to a desired displacement is designed using optimal control theory. MPC and LQR controllers are used to design and improve the performance of the hydraulic actuator. The hydraulic actuator system is linearized using Taylor series linearization method and designed using Matlab/Simulink tool. Comparison of the hydraulic actuator with MPC and LQR controllers using three desired output displacement signals (step, sine wave and white noise) is done and simulation results have been analyzed successfully. For the desired step input signal, the hydraulic actuator system with MPC controller lower rise and settling times with small percentage overshoot as compared to the hydraulic actuator system with LQR controller and for the desired sine wave signal, the hydraulic actuator system with MPC controller almost track the desired sine wave input signal correctly as compared to the hydraulic actuator system with LQR controller. While for the desired white noise input signal, the hydraulic actuator system with MPC controller have tried to track the desired white noise input signal with small variation in amplitude as compared to the hydraulic actuator system with LQR controller. Finally the comparative simulation results prove the effectiveness of the proposed hydraulic actuator system with MPC controller.
\end{abstract}

Keywords: Hydraulic actuator, Model predictive control (MPC), linear quadratic regulator (LQR)

\section{Introduction}

Hydraulic actuators get their power from pressurized hydraulic fluid, which is commonly oil. The hydraulic actuator includes a cylinder barrel, wherein a piston connected to a piston rod moves from side to side. The barrel is closed on one cease through the cylinder backside (additionally known as the cap) and the opposite give up by the cylinder head (additionally referred to as the gland) in which the piston rod comes out of the cylinder. The piston has sliding gold and seals. The piston divides the inner of the cylinder into two chambers, the lowest chamber (cap end) and the piston rod facet chamber (rod cease / head quit). Flanges, trunnions, clevises, and lugs are common cylinder mounting options. The piston rod also has mounting attachments to attach the cylinder to the item or system aspect that it is pushing or pulling.

A hydraulic actuator is the motor side of this system. The "generator" facet of the hydraulic system is the hydraulic pump which can provide a hard and fast or regulated go with the flow of oil to the 
hydraulic cylinder, to transport the piston. The piston pushes the oil within the other chamber back to the reservoir. If the oil enters from cap stop, all through extension stroke, and the oil strain in the rod end / head stop is about 0 , the force $\mathrm{F}$ on the piston rod equals the pressure $\mathrm{P}$ in the cylinder instances the piston area A.

\section{Mathematical Modelling}

The hydraulic actuator is shown in Figure 1 below. The hydraulic actuator system is fed with fluid from a constant pressure source in which the compressibility of the fluid is negligible.

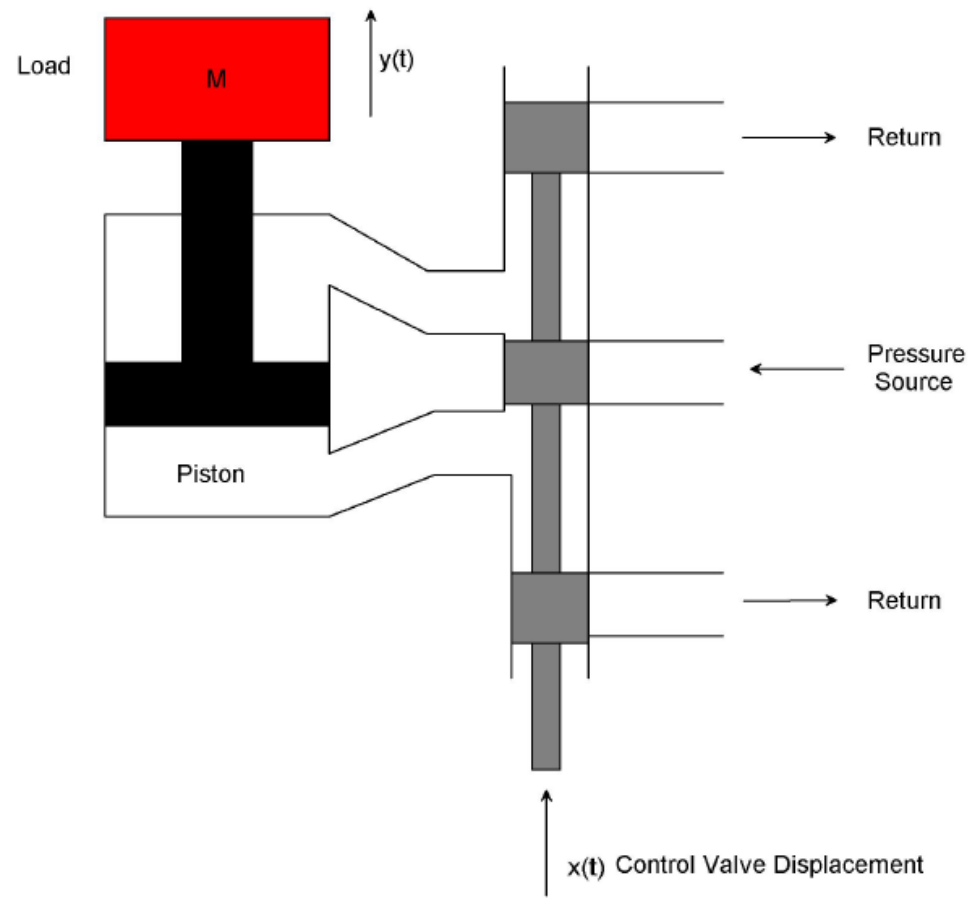

Figure 1 the hydraulic actuator system

An upward input displacement x moves the control valve; thus fluid passes in to the upper part of the cylinder and the piston is forced upward. A low power displacement of $\mathrm{x}(\mathrm{t})$ causes a large high power displacement $y(t)$. The flow rate of the fluid $Q$ is related to the input displacement $x(t)$ and the differential pressure across the piston will be

$$
Q=g(x, P)
$$

Using Taylor series linearization technique we have

$$
Q=\left(\frac{\partial g}{\partial x}\right)_{x_{0}, p_{0}} x+\left(\frac{\partial g}{\partial P}\right)_{x_{0}, p_{0}} P=k_{x} x+k_{p} P
$$

Where $g=g(x, P)$ and $\left(x_{0}, p_{0}\right)$ is the operating point, the piston developed a force which is the area of the piston A multiplied by the pressure $\mathrm{P}$. 
The applied force to the mass become

$$
A P=M \frac{d^{2} y}{d t^{2}}+b \frac{d y}{d t}
$$

Where

M mass of the lifted body

b Damping coefficient of the piston

Substituting Equation 2 in to Equation 3 yields:

$$
\frac{A}{k_{p}}\left(k_{x} x-Q\right)=M \frac{d^{2} y}{d t^{2}}+b \frac{d y}{d t}
$$

The fluid flow is related to the piston movement as

$$
Q=A \frac{d y}{d t}
$$

Substituting Equation 5 in to Equation 4 and rearranging yields:

$$
\frac{A k_{x}}{k_{p}} x=M \frac{d^{2} y}{d t^{2}}+\left(b+\frac{A^{2}}{k_{p}}\right) \frac{d y}{d t}
$$

Therefore the transfer function between the output displacement and input displacement will be:

$$
\frac{Y(s)}{X(s)}=\frac{K}{s(M s+D)}
$$

Where

$$
K=\frac{A k_{x}}{k_{p}} \text { and } D=b+\frac{A^{2}}{k_{p}}
$$

The hydraulic actuator parameters is shown in Table 1 below

Table 1 Hydraulic actuator parameters

\begin{tabular}{|l|l|l|l|}
\hline No & Parameters & Symbol & Value \\
\hline 1 & Mass of the body & M & $3 \mathrm{~kg}$ \\
\hline 2 & Area of the piston & A & $0.5 \mathrm{~m} 2$ \\
\hline 3 & Damping coefficient of the piston & b & $9.9375 \mathrm{~N}-\mathrm{s} / \mathrm{m}$ \\
\hline 4 & Displacement constant & $k_{x}$ & 8 \\
\hline 5 & Pressure constant & $k_{p}$ & 4 \\
\hline
\end{tabular}

The transfer function numerically will be 


$$
\frac{Y(s)}{X(s)}=\frac{1}{s(3 s+10)}
$$

The state space representation of the hydraulic actuator will be:

$$
\begin{aligned}
& \dot{x}=\left(\begin{array}{cc}
-3.3333 & 0 \\
1 & 0
\end{array}\right) x+\left(\begin{array}{l}
1 \\
0
\end{array}\right) u \\
& y=\left(\begin{array}{ll}
0 & 0.3333
\end{array}\right) x
\end{aligned}
$$

\section{The Proposed Controllers Design}

3.1 LQR Control

$\mathrm{LQR}$ is a manipulate scheme that gives the pleasant feasible performance with respect to a few given degree of performance. The overall performance degree is a quadratic feature composed of state vector and manage input. Linear Quadratic Regulator (LQR) is the top of the line principle of pole placement method. LQR algorithm defines the gold standard pole place primarily based on two main feature. To find the finest gains, one need to define the most advantageous performance index first of all and then clear up algebraic Riccati equation. LQR does now not have any unique strategy to outline the value feature to attain the most suitable gains and the cost function need to be described in iterative manner.

LQR is a manipulate scheme that offers the quality feasible overall performance with respect to a few given measure of overall performance. The LQR design hassle is to design a state comments controller $\mathrm{K}$ such that the index performance $\mathrm{J}$ is minimized. In this method a remarks gain matrix is designed which minimizes the objective feature so as to achieve some compromise between the usage of control attempt, the importance, and the speed of reaction a good way to assure a stable system. Designer pick the correct cost of $\mathrm{Q}$ and $\mathrm{R}$ to discover the perfect benefit matrix $\mathrm{K}$ the usage of MATLAB. The state variable configuration is shown below in Figure 2.

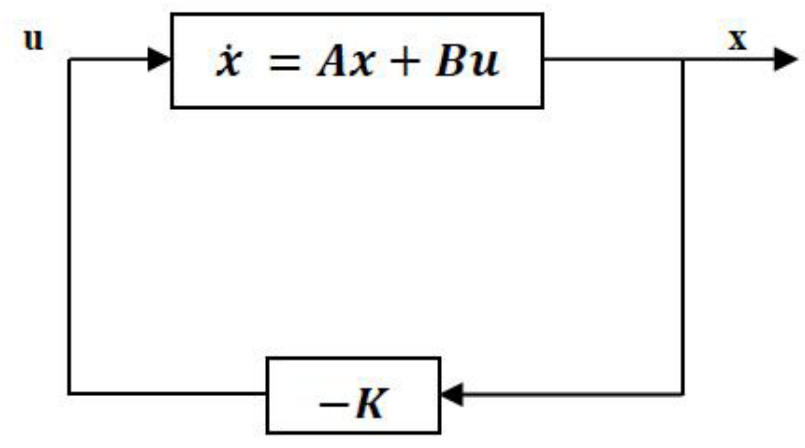

Figure 2 State variable feedback configuration

In this paper, the value of $\mathrm{Q}$ and $\mathrm{R}$ is chosen as

$$
Q=\left(\begin{array}{ll}
5 & 0 \\
0 & 5
\end{array}\right) \text { and } R=10
$$


The value of obtained feedback gain matrix $K$ of $L Q R$ is given by

$$
K=\left(\begin{array}{ll}
0.2757 & 0.7071
\end{array}\right)
$$

\subsection{MPC Control}

A block diagram of a model predictive control system is shown in Figure 3. A system model is used to count on the modern values of the output variables.

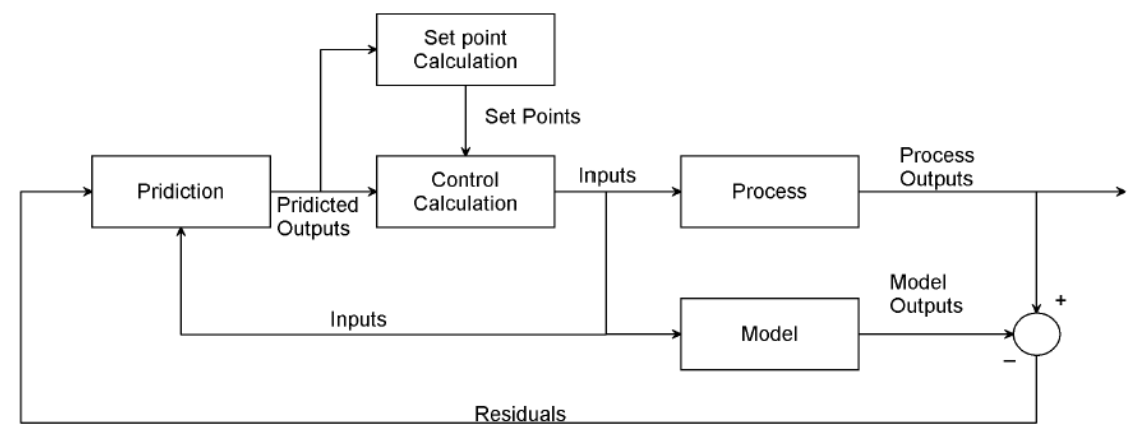

Figure 3 Block diagram for model predictive control.

The residuals, the variations a number of the actual and predicted outputs, function the comments sign to a Prediction block. The predictions are utilized in two styles of MPC calculations which might be achieved at each sampling right now: set-factor calculations and manage calculations. Inequality constraints at the input and output variables, consisting of better and decrease limits, can be included in both type of calculation.

\section{Result and Disscusion}

Here in this section, the comparison of hydraulic actuator system with MPC and LQR controllers analysis, simulation and result is done.

\subsection{Comparison of the hydraulic actuator system with MPC and LQR controllers for a step Desired Input}

The simulink model for the hydraulic actuator system with MPC and LQR controllers for a step desired input signal is shown in Figure 4 below. 


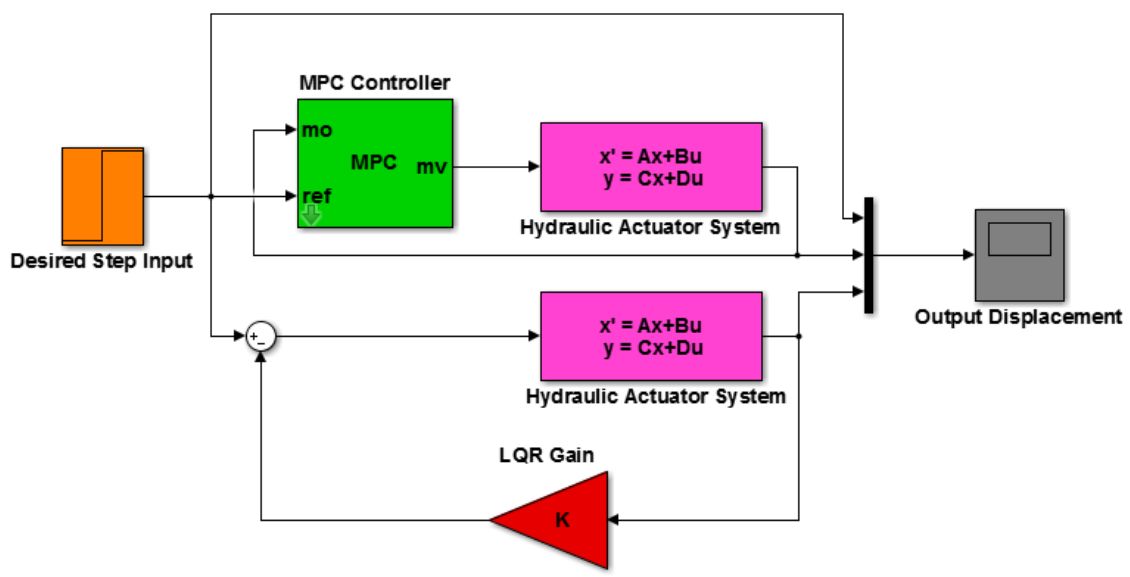

Figure 4 simulink model for the hydraulic actuator system with MPC and LQR controllers for a step desired input signal

The simulation result of the hydraulic actuator system with MPC and LQR controllers for a step desired input signal is shown in Figure 5 below.

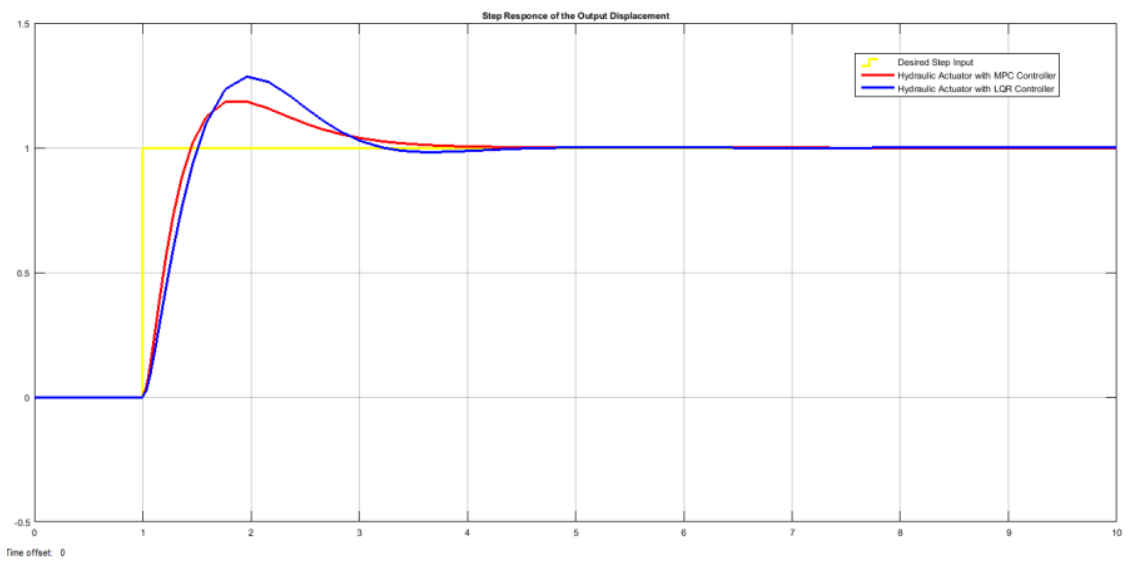

Figure 5 simulation result of the hydraulic actuator system with MPC and LQR controllers for a step desired input signal

As Figure 5 result, the hydraulic actuator system with MPC controller lower rise and settling times with small percentage overshoot as compared to the hydraulic actuator system with LQR controller.

\subsection{Comparison of the hydraulic actuator system with MPC and LQR controllers for a Sine Wave Desired Input}

The simulink model for the hydraulic actuator system with MPC and LQR controllers for a sine wave desired input signal is shown in Figure 6 below. 


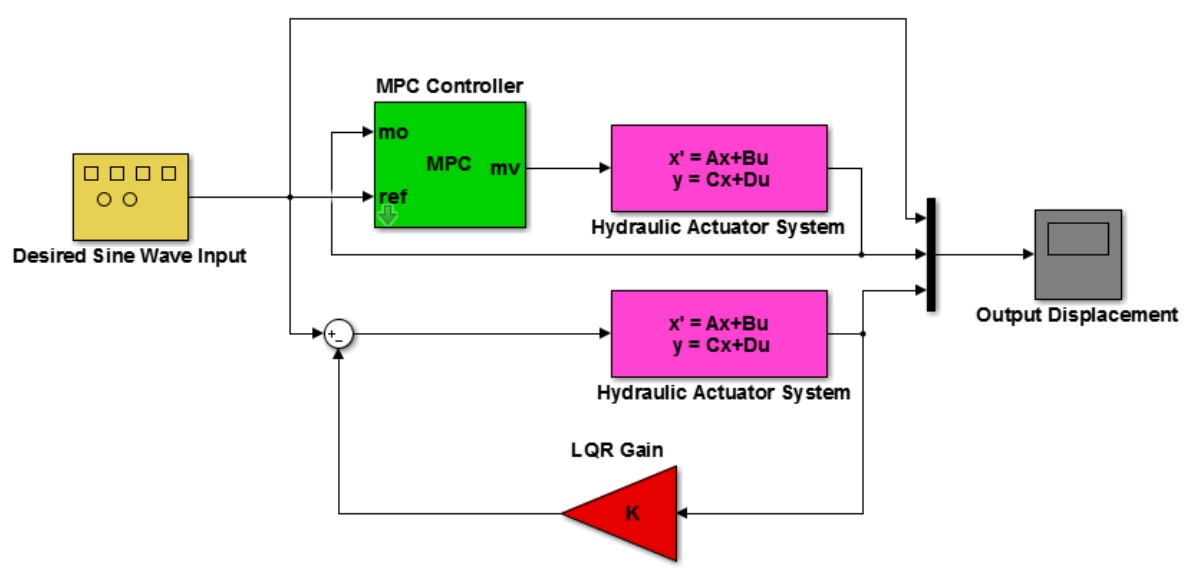

Figure 6 simulink model for the hydraulic actuator system with MPC and LQR controllers for a sine wave desired input signal

The simulation result of the hydraulic actuator system with MPC and LQR controllers for a sine wave desired input signal is shown in Figure 7 below.

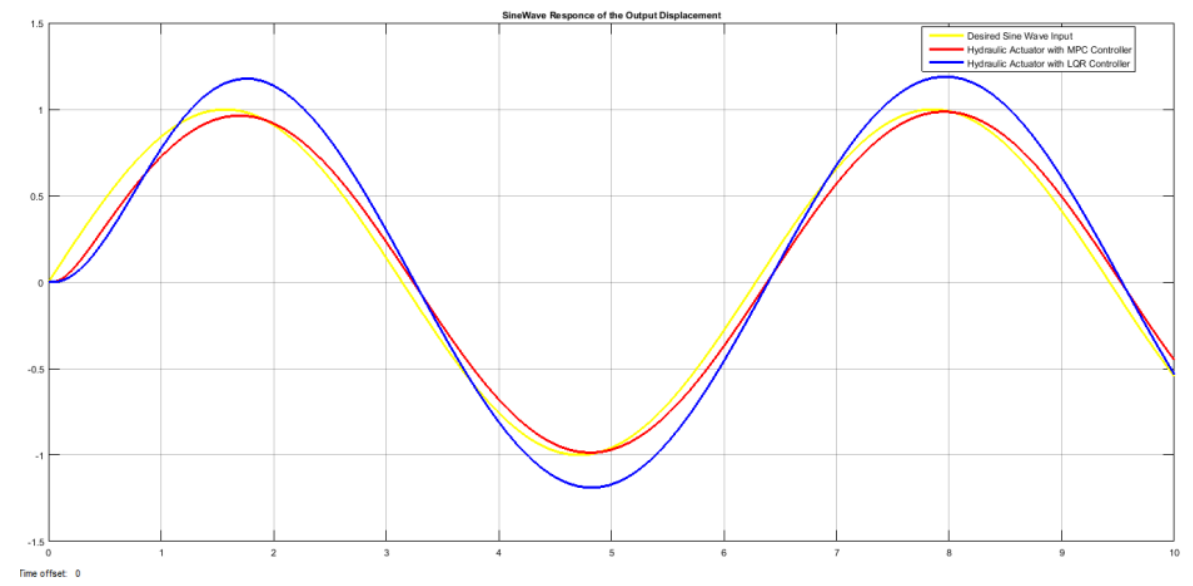

Figure 7 simulation result of the hydraulic actuator system with MPC and LQR controllers for a sine wave desired input signal

As Figure 7 result, the hydraulic actuator system with MPC controller allmost track the desired sine wave input signal correctlyas compared to the hydraulic actuator system with LQR controller.

\subsection{Comparison of the hydraulic actuator system with MPC and LQR controllers for a White Noise Desired Input}

The simulink model for the hydraulic actuator system with MPC and LQR controllers for a white noise desired input signal is shown in Figure 8 below. 


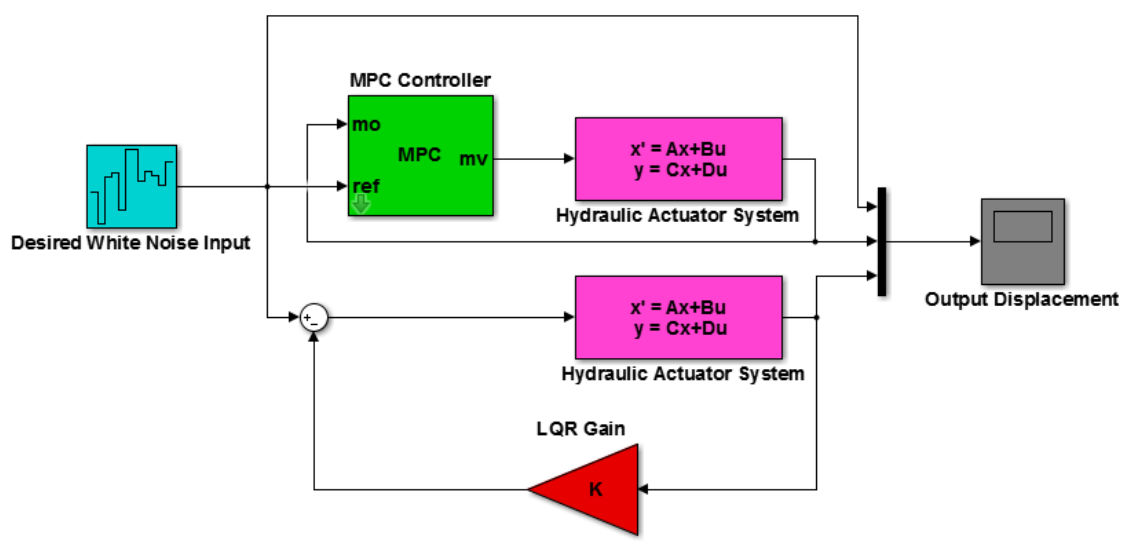

Figure 8 simulink model for the hydraulic actuator system with MPC and LQR controllers for a white noise desired input signal

The simulation result of the hydraulic actuator system with MPC and LQR controllers for a white noise desired input signal is shown in Figure 9 below.

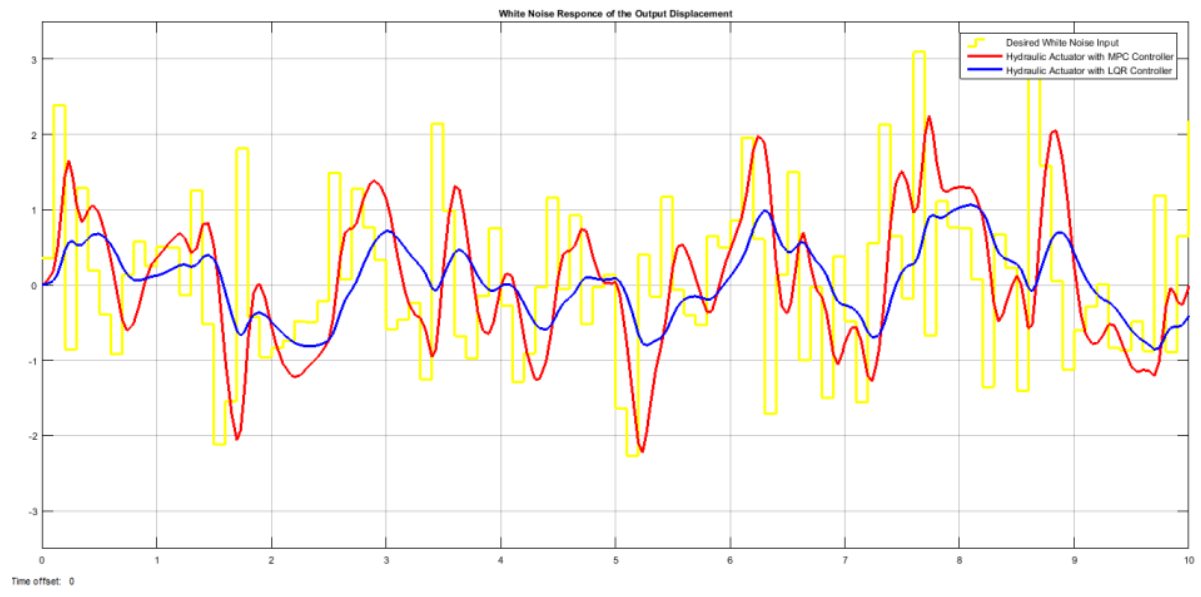

Figure 9 simulation result of the hydraulic actuator system with MPC and LQR controllers for a white noise desired input signal

As Figure 9 result, the hydraulic actuator system with MPC controller have tried to track the desired white noise input signal with small variation in amplitude as compared to the hydraulic actuator system with LQR controller.

\section{Conclusion}

The hyraulic actuator mass lifter system using MPC and LQR controllers comparison and analysis is done with the help of Matlab/Simulink toolbox succesfully. Comparison of the hydraulic actuator with MPC and LQR controller is done by tracking a desired output displacement for three input signals. The simulation results shows that the hydraulic actuator with MPC controller improve the system performance in the three input signals. Finally the comparative simulation results prove the effectiveness of the proposed hydraulic actuator system with MPC controller. 
This system is designed for a small mass lifting system, so for the future scope this system can be designed for heavy masses with the help of microcontrollers to make the system performance more accurate.

\section{Reference}

[1]. Tongil Park et al. "Electro-Hydraulic Actuator for a Soft Gripper" Soft Robotics, Vol. 7, No. 1, 2020.

[2]. Manisha et al. "Finite Element Analysis of Hydraulic Actuator by using CAE Tools" International Journal of Engineering Research \& Technology, Vol. 09, Issue. 03, 2020.

[3]. Aida Parvaresh et al. " Model Predictive Control of a Hydraulic Actuator in Torque Applying System of a Mechanically Closed Loop Test Rig for the Helicopter Gearbox" VGTU Journals, Vol. 23, No. 4, 2019.

[4].G Karthik et al. "Design \& Analysis of Hydraulic Actuator in a Typical Aerospace Vehicle” Journal for Research, Vol. 04, Issue. 02, 2018.

[5].Juliang X. et al. "Theoretical and Experimental Analysis of the Hydraulic Actuator used in the Active Reflector System" Mathematical Problems in Engineering, Article ID 8503628, 13 pages, 2018.

[6]. Guttilokesh "Electro Hydraulic Actuator Control and Operation" International Journal of Mechanical Engineering, Vol. 5, Issue. 4, 2018.

[7].N Ishak et al. "Modelling and Controller Design on ARX Model of Electro-Hydraulic Actuator" Journal of Fundamental and Applied Sciences, Vol. 9, No. 4s, Special Issue, 2017.

[8].D R S Raghuraman et al. "Simulation of Proportional Control of Hydraulic Actuator using Digital Hydraulic Valves" IOP Conference Series: Material Science and Engineering, Vol. 263, Issue. 6, 2017.

[9].S Salleh et al. "Review on Modelling and Controller Design of Hydraulic Actuator Systems" International Journal on Smart Sensing and Intelligent Systems, Vol. 8, Issue. 1, pp. 338-367, 2015. 